\title{
Identification of oil-film coefficients for a rotor-journal bearing system based on equivalent load reconstruction
}

Kun $\mathrm{Li}^{\text {a }}$, Jie Liu*, a ${ }^{*}$ Xu Han ${ }^{\text {a }}$, Chao Jiang ${ }^{\text {a }}$, Hongjun Qin ${ }^{\mathrm{b}}$

a State Key Laboratory of Advanced Design and Manufacturing for Vehicle Body, College of Mechanical and Vehicle Engineering, Hunan University, Changsha 410082, P.R. China

${ }^{\mathrm{b}}$ CZ-HNU Institute of Machinery Equipment

*Corresponding author.

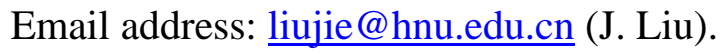




\begin{abstract}
In this paper, a laboratory method based on equivalent dynamic load reconstruction is proposed for the identification of oil-film coefficients. When modeling the rotor, the oil-film supports are considered as its dynamic load boundary conditions. Consequently, the identification of oil-film coefficients is first converted to the reconstruction of equivalent dynamic loads. Through Green's function method and regularization, the equivalent dynamic oil-film loads can be steadily and precisely reconstructed. Then, according to the mechanical relationships between the oil-film properties and the corresponding equivalent loads, the oil-film stiffness and damping coefficients are identified using least square scheme. A rotor structure with two journal bearings is investigated and the identification results of oil-film coefficients demonstrate the validity and accuracy of the proposed method.
\end{abstract}

Keywords: Oil-film coefficients, load identification, rotor-journal bearing system, Green's function method 


\section{Introduction}

Rotor-journal bearing systems have wide applications in rotating machines, and have attracted much interest from engineers and researchers to analyze their characteristics and performances. As the rotor rotates at a high speed with the direct support from the oil-film of journal bearing, the oil-film clearance between rotor and journal bearing will get changed when the rotor slightly deviates from its original equilibrium position. This change causes direct alterations on the oil-film support effects, and further affects the unbalance response of the rotor and the system stability performance, etc. In this process, all the alterations are closely related to the dynamic oil-film properties of journal bearing, which can usually be represented by linearized dynamic stiffness and damping coefficients. Thus, to obtain the reliable characteristic coefficients of oil-film is particularly important for understanding the dynamic behavior of the rotor-journal bearing system. But unfortunately, as the limits of technical conditions and numerous factors, it is really difficult or impossible for the direct experimental measurement of the oil-film properties. Therefore, to develop indirect identification approaches for the oil-film coefficients become quite necessary and valuable.

So far many relevant indirect identification methods have already been proposed [14] based on the rotor-bearing system and corresponding dynamic measurements. Among them, the impulse response-based method is one of the earliest and most widely studied methods, as an impulse excitation covers a wide range of frequency characteristics, which increases the reliability of identified coefficients. For example, Nicholas and Gunter [5] identified the oil-film stiffness and damping parameters of two symmetric bearings by the curve fitting of frequency responses due to impact excitation. Tieu and Qiu $[6,7]$ respectively utilized experimental and calculated impulse responses of rotor to determine the bearing characteristics which simplified the coefficient 
calculation by the synchronous unbalance responses. Jiang and $\mathrm{Hu}[8]$ attempted to add measuring facilities to investigate the dynamic oil-film characteristics of different bearings on a large full scale journal bearing rig, which took the impulse excitation, vibration measurement, data $\log$ and dynamic characteristics identification into consideration, and met the need of stability study. Tiwari et al. $[9,10]$ investigated the simultaneous identifications of the residual unbalances and dynamic bearing coefficients through two separate identification methods using impulse responses from the bearing housings and unbalance responses from three different unbalance configurations, respectively. Meanwhile, there were also many other investigations on the identification of oil-film coefficients. Zhang and Xie [11] identified the linearized oil-film coefficients by fitting the measured frequency response functions to the theoretically calculated ones. Zhao et al. $[12,13]$ proposed three kinds of nonlinear oilfilm load models by retaining certain terms of Taylor series expansion of the oil-film load, and identified the oil-film coefficients by least-mean-square method in time domain. Makowski and Zimroz [14] proposed a procedure for feature extraction using adaptive Schur filter for damage detection in rolling element bearings, in which the local change of stiffness related with the impact shocks was studied. Zhang et al. [15] provided a method for determining the stiffness coefficients of hydrodynamic plain journal bearings lubricated by water. It considered the cavitation effect of water and obtained the relationships between the stiffness coefficients and the load for bearings with different relative clearances and different rotate speeds. Wang et al. [16] combined the rational polynomial method with the weighted instrumental variable estimator to fit the directional frequency response function and identified the damping ratio of the first forward and backward modes of rotor systems. Zhou et al. [17] estimated the closedloop stiffness and damping coefficients of active magnetic bearings (AMB) using the 
unbalance responses of rotor, and validated the identification results with a flexible rotor-AMB test rig. Zhou et al. [18] performed an experimental study to recognize the bearing parameters and establish their features under varieties of operation conditions, and found by experiments that the linear oil-film dynamic coefficients were sensitive to the excitation load. Synnegård et al. [19] evaluated how cross-coupling coefficients affects the dynamics of a vertical rotor with tilting pad journal bearings, demonstrating that the cross-coupling stiffness and damping coefficients should be included when simulating vertical machines subjected to high loads. Kim and Yang [20] acquired not only the bearing parameters but also the unknown information of the disc based on the clustering-based hybrid optimization algorithm using measured unbalance response. Meruane and Pascual [21] numerically calculated the nonlinear parameters of fluid film which were defined by a third order Taylor expansion of bearing reaction loads from large journal orbital motion, and evaluated them through a least mean square in time domain. For the above mentioned methods, in order to achieve the identification of oilfilm coefficients, either the numerical models of both rotor and journal bearing should be simultaneously established in advance, or the transform relationships related with the oil film should be deduced. However, as many factors (such as pressure boundary conditions, temperature boundary conditions, cavitations, etc.) have important influences on the system performances, and the contact between oil-film and rotor is complex and difficult to be simulated, it is really difficult to build a reasonable and accurate numerical or analytical model for rotor-bearing system. Even with the obtained models, researchers have reported that often the regression matrix of the estimation equation became ill-conditioned, and this led to scattering of the identified oil-film parameters [12]. 
Though the accurate model of the rotor-journal bearing system is difficult to be obtained, the separate rotor structure is simple and can be accurately modeled through finite element techniques. By considering the oil-film supports as dynamic load boundary conditions on the rotor, the complicated modeling of the oil-film can be avoided. In this view, this paper presents a new method for the identification of oil-film coefficients of a rotor-journal bearing system. It converts the identification problem of oil-film coefficients to the reconstruction problem of equivalent dynamic loads for the easy modeling rotor structure. Green's function method with regularization and least square scheme are adopted to realize the stable and accurate identification of oil-film coefficients. The remainder of this paper is organized as follows: In Section 2, the complicated rotor-journal bearing system is simplified as a rotor structure with the supporting condition of unknown equivalent oil-film loads. In Section 3, the equivalent loads are precisely reconstructed using Green's function method and regularization, and subsequently, the oil-film coefficients are obtained by least square scheme. In Section 4, a numerical example is provided to reveal the identification process and validate the correctness of the identified oil-film coefficients. Some concluding remarks are made in Section 5.

\section{Equivalence of oil-film load}

The steady-state kinetic equation of a rotor-journal bearing system under unbalance load can be expressed as $[8,9,22]$

$$
\mathbf{M} \ddot{\mathbf{q}}(t)+(\omega \boldsymbol{\Omega}+\mathbf{C}) \dot{\mathbf{q}}(t)+\mathbf{K q}(t)=\mathbf{F}^{\mathrm{U}}(t)
$$

where $\mathbf{M}, \boldsymbol{\Omega}, \mathbf{C}$ and $\mathbf{K}$ respectively represent the whole structural mass, gyroscopic, damping and stiffness matrices. $\ddot{\mathbf{q}}(t), \dot{\mathbf{q}}(t)$ and $\mathbf{q}(t)$ are the acceleration, velocity and displacement unbalance response vectors. $\omega$ denotes the 
angular speed of rotor and $\mathbf{F}^{\mathrm{U}}(t)$ is the periodic unbalance load. For expression simplicity in the following statement, we first take the case with single unbalance mass and single journal bearing without loss of generality.

Considering the rotor unbalance mass rotates with the rotor speed, the unbalance load generated by unbalance mass equal to

$$
\left[\begin{array}{c}
F_{x}^{\mathrm{U}}(t) \\
F_{y}^{\mathrm{U}}(t)
\end{array}\right]=m e \omega^{2}\left[\begin{array}{c}
\sin (\omega t+\varphi) \\
\cos (\omega t+\varphi)
\end{array}\right]
$$

where $F_{x}^{\mathrm{U}}(t)$ and $F_{y}^{\mathrm{U}}(t)$ are respectively the horizontal and vertical radial components of unbalance load. $m$ is the unbalance mass of rotor, $e$ and $\varphi$ are the eccentricity and initial phase. As the finite element matrices based on Timoshenko beam [23] are defined in terms of the rotor elasto-massic properties, the unbalance response vector of rotor is

$$
\mathbf{q}=\left[\begin{array}{llll}
\mathbf{q}_{1} & \mathbf{q}_{2} & \cdots & \mathbf{q}_{N}
\end{array}\right]^{\mathrm{T}} ; \mathbf{q}_{i}=\left[\begin{array}{llll}
\mu_{x} & \mu_{y} & \theta_{x} & \theta_{y}
\end{array}\right]^{\mathrm{T}}, i=1,2, \cdots, N
$$

where $N$ is the rotor's node number, $\left(\mu_{x}, \mu_{y}\right)$ and $\left(\theta_{x}, \theta_{y}\right)$ respectively represent the lateral displacements and rotations of the rotor motion coordinates. The global mass $\mathbf{M}$, stiffness $\mathbf{K}_{R}$, damping $\mathbf{C}_{R}$, and gyroscopic $\boldsymbol{\Omega}$ matrices of the separate rotor can be assembled as

$$
\mathbf{M}=\bigcup_{i=1}^{N} \mathbf{M}_{i}, \mathbf{C}_{R}=\bigcup_{i=1}^{N} \mathbf{C}_{i}, \mathbf{K}_{R}=\bigcup_{i=1}^{N} \mathbf{K}_{i}, \boldsymbol{\Omega}=\bigcup_{i=1}^{N} \boldsymbol{\Omega}_{i}
$$

And therefore, the damping matrix $\mathbf{C}$ and stiffness matrix $\mathbf{K}$ in Eq. (1) combine the effects of the rotor and the oil-film supports of bearing, namely,

$$
\mathbf{C}=\mathbf{C}_{R}+\left[\begin{array}{ccc}
\mathbf{0} & \cdots & \cdots \\
\vdots & \mathbf{C}_{B} & \vdots \\
\cdots & \cdots & \mathbf{0}
\end{array}\right], \mathbf{K}=\mathbf{K}_{R}+\left[\begin{array}{ccc}
\mathbf{0} & \cdots & \cdots \\
\vdots & \mathbf{K}_{B} & \vdots \\
\cdots & \cdots & \mathbf{0}
\end{array}\right]
$$

where $\mathbf{C}_{B}$ and $\mathbf{K}_{B}$ represent the oil-film damping and stiffness matrices, 
respectively. As shown in Fig. 1, the oil-film supports can be generally approximated with four linearized damping and stiffness coefficients [18], so $\mathbf{C}_{B}$ and $\mathbf{K}_{B}$ are of the forms as

$$
\mathbf{C}_{B}=\left[\begin{array}{ll}
c_{x x} & c_{x y} \\
c_{y x} & c_{y y}
\end{array}\right], \mathbf{K}_{B}=\left[\begin{array}{ll}
k_{x x} & k_{x y} \\
k_{y x} & k_{y y}
\end{array}\right]
$$

where $c_{x x}, c_{x y}, c_{y x}, c_{y y}, k_{x x}, k_{x y}, k_{y x}, k_{y y}$ are exactly the oil-film characteristic coefficients to be identified. Further, according to the dynamic theory, the equivalent oil-film loads can be set up with the following relationships

$$
\left[\begin{array}{c}
F_{x}^{\mathrm{E}}(t) \\
F_{y}^{\mathrm{E}}(t)
\end{array}\right]=\left[\begin{array}{cc}
c_{x x} & c_{x y} \\
c_{y x} & c_{y y}
\end{array}\right]\left[\begin{array}{c}
\dot{\mu}_{x}(t) \\
\dot{\mu}_{y}(t)
\end{array}\right]_{B}+\left[\begin{array}{cc}
k_{x x} & k_{x y} \\
k_{y x} & k_{y y}
\end{array}\right]\left[\begin{array}{l}
\mu_{x}(t) \\
\mu_{y}(t)
\end{array}\right]_{B}
$$

where $F_{x}^{\mathrm{E}}(t)$ and $F_{y}^{\mathrm{E}}(t)$ respectively denote the horizontal and vertical radial oilfilm component load, the index $B$ denotes the location of the bearing support. It is notable from Eq. (7) that once the oil-film loads $\left[\begin{array}{c}F_{x}^{\mathrm{E}}(t) \\ F_{y}^{\mathrm{E}}(t)\end{array}\right]$, and the velocities $\left[\begin{array}{c}\dot{\mu}_{x}(t) \\ \dot{\mu}_{y}(t)\end{array}\right]_{B}$ and displacements $\left[\begin{array}{l}\mu_{x}(t) \\ \mu_{y}(t)\end{array}\right]_{B}$ are obtained, the eight oil-film coefficients can be identified by the inverse calculation of Eq. (7).

$$
\text { Denoting } \quad \mathbf{F}^{\mathrm{E}}(t)=\left[\begin{array}{llllllll}
0 & \cdots & F_{x}^{\mathrm{E}}(t) & F_{y}^{\mathrm{E}}(t) & 0 & 0 & \cdots & 0
\end{array}\right]^{\mathrm{T}} \text {, and moving } \mathbf{F}^{\mathrm{E}}(t)
$$

from the left side of Eq. (1) to the right side, the following equivalent kinetic equation of the rotor structure subjecting to loads $\mathbf{F}^{\mathrm{U}}(t)$ and $-\mathbf{F}^{\mathrm{E}}(t)$ can be obtained,

$$
\mathbf{M} \ddot{\mathbf{q}}(t)+\left(\omega \boldsymbol{\Omega}+\mathbf{C}_{R}\right) \dot{\mathbf{q}}(t)+\mathbf{K}_{R} \mathbf{q}(t)=\mathbf{F}^{\mathrm{U}}(t)-\mathbf{F}^{\mathrm{E}}(t)
$$

With Eq. (8), the identification of oil-film coefficients is indirectly transformed into the reconstruction of the equivalent oil-film loads, which completely avoids the oil-film modeling. Furthermore, for the case with multi unbalance masses and journal bearings, 
Eq. (8) can be achieved as well with $\mathbf{F}^{\mathrm{U}}(t)$ and $\mathbf{F}^{\mathrm{E}}(t)$ correspondingly containing multi components. In the following section, the equivalent load is to be reconstructed by the Green's function method and regularization operation and then combining Eq. (7), the oil-film coefficients will be inversely identified.

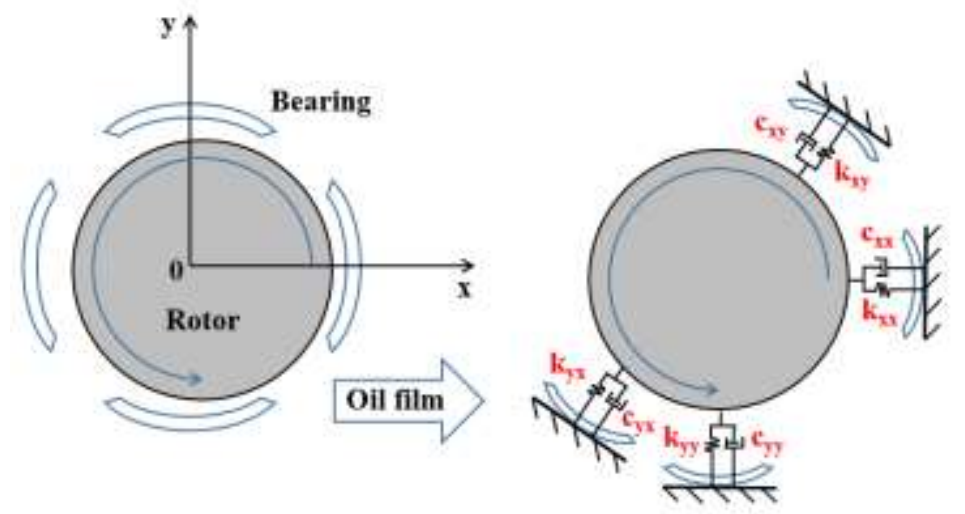

Fig. 1 Schematic view of rotor on bearing supports and idealization of oil-film coefficients

\section{Identifications of equivalent oil-film loads and oil-film coefficients}

Considering Eq. (8), as the rotor structure is linear time invariant, the measurable response $\mathbf{q}(t)$ can be thought to be the superposition of responses of the rotor separately subjecting to $\mathbf{F}^{\mathrm{U}}(t)$ and $-\mathbf{F}^{\mathrm{E}}(t)$. Hence, the following relationship is obtained according to the superposition principle,

$$
\mathbf{M} \ddot{\mathbf{q}}^{\mathrm{E}}(t)+\left(\omega \boldsymbol{\Omega}+\mathbf{C}_{R}\right) \dot{\mathbf{q}}^{\mathrm{E}}(t)+\mathbf{K}_{R} \mathbf{q}^{\mathrm{E}}(t)=\mathbf{F}^{\mathrm{E}}(t)
$$

where

$$
\mathbf{q}^{\mathrm{E}}(t)=\mathbf{q}^{\mathrm{U}}(t)-\mathbf{q}(t)
$$

$\mathbf{q}^{\mathrm{E}}(t)$ and $\mathbf{q}^{\mathrm{U}}(t)$ respectively represent the dynamic response of the rotor structure only subjecting to the equivalent oil-film load $\mathbf{F}^{\mathrm{E}}(t)$ and the unbalance load $\mathbf{F}^{\mathrm{U}}(t)$. As the matrices $\mathbf{M}, \mathbf{K}_{\mathrm{R}}, \mathbf{C}_{\mathrm{R}}$, and $\boldsymbol{\Omega}$ are available, and the unbalanced load $\mathbf{F}^{\mathrm{U}}(t)$ can be easily obtained with the information of the unbalance mass, the unbalance response $\mathbf{q}^{\mathrm{U}}(t)$ can be numerically and accurately calculated. Besides, the structural 
response $\mathbf{q}(t)$ can be obtained via measurement, so the response $\mathbf{q}^{\mathrm{E}}(t)$ for the identification of equivalent oil-film loads is also available.

\subsection{Reconstruction of equivalent oil-film load}

In general, the equivalent dynamic load $\mathbf{F}^{\mathrm{E}}(t)$ in Eq. (9) is multi-source. For the actual case that the rotor is supported by $H$ journal bearings, there will be $Z=2 H$ equivalent oil-film loads. For expression convenience, we denote the horizontal radial component load $F_{x}^{\mathrm{E}}(t)$ of the $i$ th journal bearing as $\bar{F}_{2 i-1}^{\mathrm{E}}(t)$, and denote the vertical radial component load $F_{y}^{\mathrm{E}}(t)$ as $\bar{F}_{2 i}^{\mathrm{E}}(t)$. To realize the reconstruction of the multisource load $\mathbf{F}^{\mathrm{E}}(t)$, it is required to reconstruct each of the equivalent load $\bar{F}_{j}^{\mathrm{E}}(t)$, $j=1,2, \cdots, Z$. According to the sampling characteristics of impulse function, i.e., the integral of the product of any function and the impulse function equals to the function value of the impact time, any load $\bar{F}_{j}^{\mathrm{E}}(t)$ can be expressed as a superposition form of impulse functions as shown in Fig. 2.

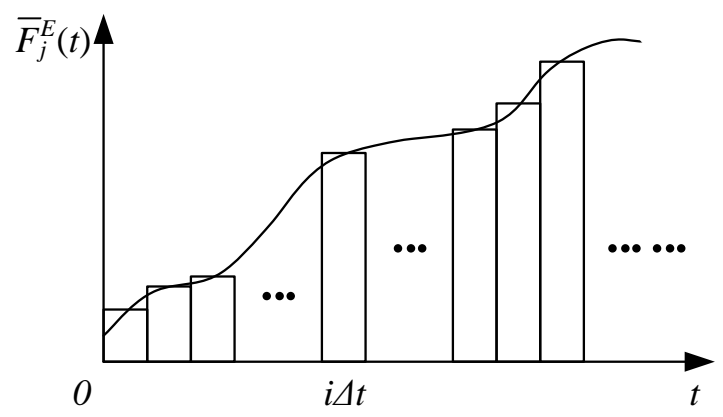

Fig. 2 Dynamic load can be expressed as the sum of the impulse functions

Accordingly, the displacement response $\mu_{i j}^{\mathrm{E}}(t)$ of the $i$ th degree of freedom (DOF) induced by the $j$ th equivalent oil-film load $\bar{F}_{j}^{\mathrm{E}}(t)$ can be expressed as the following convolution integral form [24-26],

$$
\mu_{i j}^{\mathrm{E}}(t)=\int_{0}^{t} g_{i j}(t-\tau) \bar{F}_{j}^{\mathrm{E}}(\tau) \mathrm{d} \tau
$$


where $g_{i j}(t)$ is the corresponding Green's function, namely the structural response under the impulse load. Discretizing the convolution integral (11) into $n$ equally spaced sample points in time domain, the matrix form of Eq. (11) is obtained that,

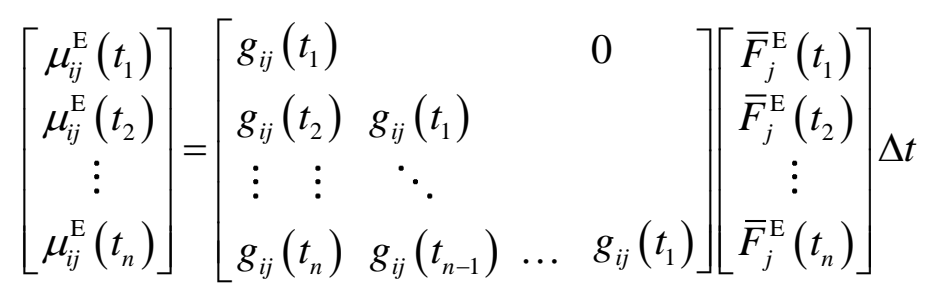

which can be simply denoted as

$$
\boldsymbol{\mu}_{i j}^{\mathrm{E}}=\mathbf{G}_{i j} \overline{\mathbf{F}}_{j}^{\mathrm{E}}
$$

Considering there are total $Z$ equivalent dynamic oil-film loads acting on the rotor, the following relationship can be obtained according to the superposition principle,

$$
\left[\begin{array}{c}
\boldsymbol{\mu}_{1}^{\mathrm{E}} \\
\boldsymbol{\mu}_{2}^{\mathrm{E}} \\
\vdots \\
\boldsymbol{\mu}_{S}^{\mathrm{E}}
\end{array}\right]=\left[\begin{array}{c}
\sum_{j=1}^{Z} \boldsymbol{\mu}_{1 j}^{\mathrm{E}} \\
Z \\
\sum_{j=1}^{\mathrm{E}} \boldsymbol{\mu}_{2 j}^{\mathrm{E}} \\
\vdots \\
Z \\
\sum_{j=1}^{Z} \boldsymbol{\mu}_{S j}^{\mathrm{E}}
\end{array}\right]=\left[\begin{array}{cccc}
\mathbf{G}_{11} & \mathbf{G}_{12} & \cdots & \mathbf{G}_{1 Z} \\
\mathbf{G}_{21} & \mathbf{G}_{22} & \cdots & \mathbf{G}_{2 Z} \\
\vdots & \vdots & \ddots & \vdots \\
\mathbf{G}_{S 1} & \mathbf{G}_{S 2} & \cdots & \mathbf{G}_{S Z}
\end{array}\right]\left[\begin{array}{c}
\overline{\mathbf{F}}_{1}^{\mathrm{E}} \\
\overline{\mathbf{F}}_{2}^{\mathrm{E}} \\
\vdots \\
\overline{\mathbf{F}}_{Z}^{\mathrm{E}}
\end{array}\right]
$$

where $S$ is the number of measured responses which is no less than $Z$ to ensure the solution is inversely computed under statically indeterminate condition.

In general, the kernel matrix of Eq. (14) established by Green's function method is ill-conditioned, and the displacement response $\boldsymbol{\mu}^{\mathrm{E}}$ calculated by Eq. (10) inevitably carries noise. Considering noise data $\boldsymbol{\mu}_{e r r}$ in the response, Eq. (14) is simply changed to the following form

$$
\boldsymbol{\mu}_{n o}^{\mathrm{E}}=\mathbf{G} \overline{\mathbf{F}}^{\mathrm{E}}+\boldsymbol{\mu}_{e r r}
$$

where $\boldsymbol{\mu}_{n o}^{\mathrm{E}}$ represents the measured structural response with noise, $\mathbf{G}$ denotes the whole Green's kernel matrix. The singular value decomposition (SVD) of $\mathbf{G}$ is of the 
form of

$$
\mathbf{G}=\mathbf{U} \Psi \mathbf{V}^{\mathrm{T}}=\sum_{i=1}^{Z \times n} \mathbf{u}_{i} \sigma_{i} \mathbf{v}_{i}^{\mathrm{T}}
$$

where $\mathbf{U}$ and $\mathbf{V}$ are the left and right singular matrices of $\mathbf{G}$ with $Z \times n$ orthonormal column vectors, $\mathbf{U}^{\mathrm{T}} \mathbf{U}=\mathbf{V}^{\mathrm{T}} \mathbf{V}=\mathbf{I}_{Z \times n}, \boldsymbol{\Psi}=\operatorname{diag}\left(\sigma_{1}, \sigma_{2} \cdots \sigma_{Z \times n}\right)$ is a diagonal matrix with nonnegative singular values arranging in a descending order. Interestingly, the decomposition of $\mathbf{G}$ gives the following estimation of the reconstructed load,

$$
\hat{\overline{\mathbf{F}}}^{\mathrm{E}}=\mathbf{G}^{-1} \boldsymbol{\mu}_{n o}^{\mathrm{E}}=\operatorname{VDiag}\left(\sigma_{i}^{-1}\right) \mathbf{U}^{\mathrm{T}} \boldsymbol{\mu}_{n o}^{\mathrm{E}}=\overline{\mathbf{F}}^{\mathrm{E}}+\sum_{i=1}^{Z \times n}\left(\mathbf{u}_{i}^{\mathrm{T}} \boldsymbol{\mu}_{e r r}\right) \mathbf{v}_{i} \sigma_{i}^{-1}
$$

Observing Eq. (17), it can be found that the errors of the estimated load are mainly caused by $\boldsymbol{\mu}_{e r r} / \sigma_{i}$. When $\sigma_{i}$ dramatically decays to zero, $\boldsymbol{\mu}_{e r r}$ is terribly magnified and the estimated load seriously deviates from the true value. Therefore, to stably and accurately reconstruct the dynamic load, it is obviously quite necessary to filter out the small singular values $\sigma_{i}$. One direct method is to multiply $\sigma_{i}$ by a regularization filter function $f_{\alpha}\left(\sigma_{i}\right)$ which contains a regularization parameter $\alpha$. As a result, the stable approximate equivalent load $\hat{\overline{\mathbf{F}}}_{\alpha}^{\mathrm{E}}$ is achieved,

$$
\hat{\overline{\mathbf{F}}}_{\alpha}^{\mathrm{E}}=\operatorname{VDiag}\left(f_{\alpha}\left(\sigma_{i}\right) \sigma_{i}^{-1}\right) \mathbf{U}^{\mathrm{T}} \boldsymbol{\mu}_{n o}^{\mathrm{E}}=\sum_{i=1}^{Z \times n} f_{\alpha}\left(\sigma_{i}\right) \sigma_{i}^{-1}\left(\mathbf{u}_{i}^{\mathrm{T}} \boldsymbol{\mu}_{n o}^{\mathrm{E}}\right) \mathbf{v}_{i}
$$

where the improved regularized filter function [27] $f_{\alpha}\left(\sigma_{i}\right)$ is adopted which has the form of

$$
f_{\alpha}\left(\sigma_{i}\right)=1-\frac{4}{\pi} \arctan \left(\exp \left(-\frac{\sigma_{i}}{\alpha}\right)\right)
$$

Additionally, the optimal regularization parameter $\alpha$ can be determined via the Generalized Cross Validation criterion (GCV) [28].

\subsection{Identification of oil-film characteristic coefficients}


From Eq. (7), it is clear that to identify the oil-film coefficients, the equivalent oilfilm loads and the corresponding velocity and displacement responses of the rotorjournal bearing structure are required. In the former part of this section, the dynamic loads are accurately reconstructed based on the Green's function method and

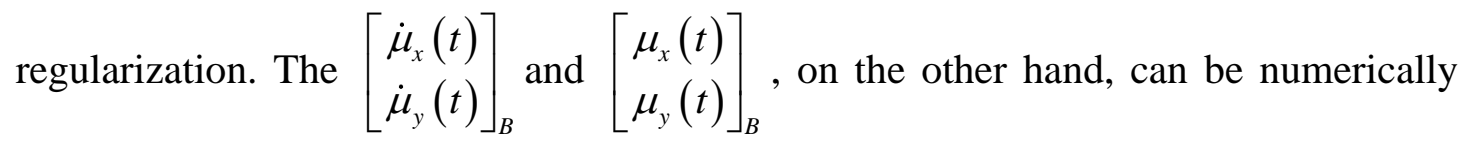
calculated by Eq. (8) as the equivalent loads have been reconstructed.

Before the coefficient identification, an analysis on the features of the horizontal and vertical radial component loads $F_{x}^{\mathrm{E}}(t)$ and $F_{y}^{\mathrm{E}}(t)$ is made for the purpose of improving the result precision. It is found that i) there is a regular $\pi / 2$ phase difference between them; ii) they have the analogous harmonic forms with the excitation of unbalance loads; iii) they disturb at the very beginning of the time series, but reach steady state quickly, which is an expected result when performing a time integration of linearized system. To comprehensively utilize the information of the equivalent oil-film loads and the corresponding responses in time domain and to reduce the influence of random errors, the weighted residual method based on least squares scheme is adopted. The residual functions are defined as follows,

$$
\left[\begin{array}{l}
R_{x}(t) \\
R_{y}(t)
\end{array}\right]=\left[\begin{array}{c}
\hat{F}_{x}^{\mathrm{E}}(t) \\
\hat{F}_{y}^{\mathrm{E}}(t)
\end{array}\right]-\left\{\left[\begin{array}{cc}
c_{x x} & c_{x y} \\
c_{y x} & c_{y y}
\end{array}\right]\left[\begin{array}{c}
\dot{\mu}_{x}(t) \\
\dot{\mu}_{y}(t)
\end{array}\right]_{B}+\left[\begin{array}{cc}
k_{x x} & k_{x y} \\
k_{y x} & k_{y y}
\end{array}\right]\left[\begin{array}{l}
\mu_{x}(t) \\
\mu_{y}(t)
\end{array}\right]_{B}\right\}
$$

Then the weighted integral of residual function in time domain is set to be zero, namely

$$
\left\{\begin{array}{l}
\int_{t_{0}}^{t} w(\tau) R_{x}(\tau) d \tau=0 \\
\int_{t_{0}}^{t} w(\tau) R_{y}(\tau) d \tau=0
\end{array}\right.
$$

where $w(t)$ is the weight function defined in time domain, $t_{0}$ determines the initial time for the integral. In this paper, $\dot{\mu}_{x}(t), \dot{\mu}_{y}(t)$ and $\mu_{x}(t), \mu_{y}(t)$ are chosen as 
the weight functions and $t_{0}$ is set the time point when the rotation curves reach stable. Now the following matrix equation for oil-film coefficient identification is obtained from Eq. (21) that

$$
\left[\begin{array}{ll}
\gamma_{11} & \gamma_{12} \\
\gamma_{21} & \gamma_{22} \\
\gamma_{31} & \gamma_{32} \\
\gamma_{41} & \gamma_{42}
\end{array}\right]=\left[\begin{array}{llll}
\eta_{11} & \eta_{12} & \eta_{13} & \eta_{14} \\
\eta_{21} & \eta_{22} & \eta_{23} & \eta_{24} \\
\eta_{31} & \eta_{32} & \eta_{33} & \eta_{34} \\
\eta_{41} & \eta_{42} & \eta_{43} & \eta_{44}
\end{array}\right]\left[\begin{array}{ll}
c_{x x} & c_{y x} \\
c_{x y} & c_{y y} \\
k_{x x} & k_{y x} \\
k_{x y} & k_{y y}
\end{array}\right]
$$

where $\gamma_{i j}=\int_{t_{0}}^{t} A_{i} B_{j} \mathrm{~d} \tau, \quad \eta_{i j}=\int_{t_{0}}^{t} A_{i} A_{j} \mathrm{~d} \tau, \quad A_{i}$ respectively corresponds to $\dot{\mu}_{x}(\tau)$, $\dot{\mu}_{y}(\tau), \mu_{x}(\tau)$ and $\mu_{y}(\tau)$ as $i=1,2,3,4, B_{i}$ respectively corresponds to $\hat{F}_{x}^{\mathrm{E}}(\tau)$ and $\hat{F}_{y}^{\mathrm{E}}(\tau)$ as $i=1,2$. Considering the Eq. (22) is positive definite, the least square scheme is adopted to achieve the accurate identification of the oil-film coefficients.

\section{Example and Discussions}

A rotor-journal bearing system with three discs and two identical journal bearings, as shown in Fig. 3a, is investigated to reveal the identification process and verify the validity of the proposed method. The geometrical parameters of the rotor-journal bearing structure are partly given in the figure. The diameters of the shaft and the discs are $40 \mathrm{~mm}$ and $100 \mathrm{~mm}$, respectively. The thickness of the discs is $30 \mathrm{~mm}$. The Young's modulus of the rotor material is $E=200 \mathrm{GPa}$, the density is $\rho=7.65 \times 10^{3} \mathrm{~kg} / \mathrm{m}^{3}$, the Poisson ratio is $\varepsilon=0.3$. The unbalance mass is located in the middle disc with $m=0.2 \times 10^{-3} \mathrm{~kg}, \quad e=40 \times 10^{-3} \mathrm{~m}$ and $\varphi=0$. The gravity is not considered in this paper for simplicity. Random noises are directly mixed into the computed responses to simulate the measured data which is defined as

$$
\mu_{n o}^{\mathrm{E}}(t)=\mu^{\mathrm{E}}(t)+L_{e r r} \operatorname{std}\left(\mu^{\mathrm{E}}(t)\right) \operatorname{rand}(-1,1)
$$


where $L_{e r r}$ is a percentage to control the noise level, $\operatorname{std}\left(\mu^{\mathrm{E}}(t)\right)$ is the standard deviation of the computed displacement response $\mu^{\mathrm{E}}(t), \operatorname{rand}(-1,1)$ is a random function with its values locating in $[-1,1]$.

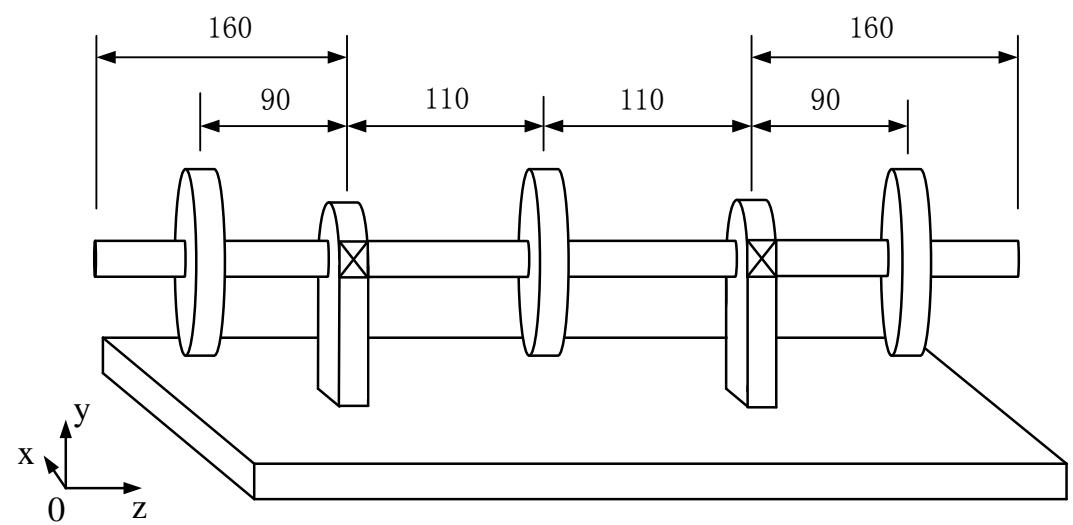

Fig. 3a Schematic view of the rotor-journal bearing structure (unit: $\mathrm{mm}$ )

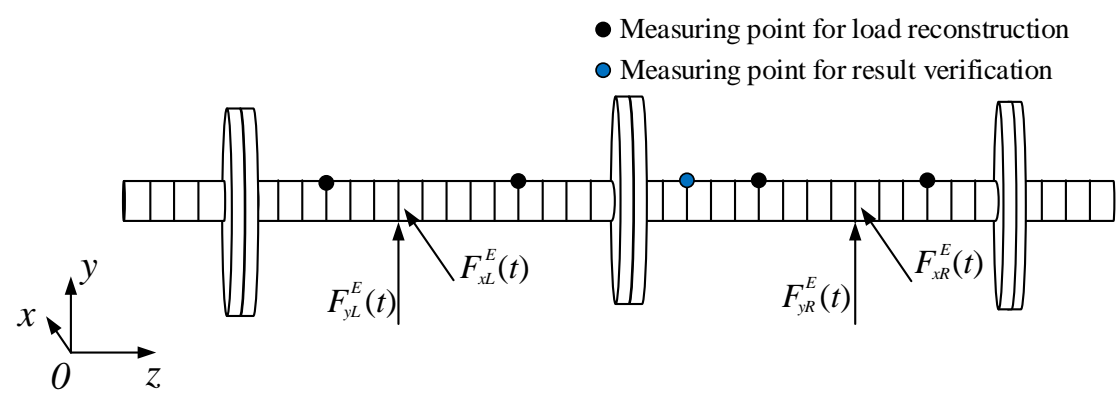

Fig. 3b The equivalent finite element model of the rotor structure

Describing the oil-film support effects of journal bearings as equivalent dynamic loads, the rotor-journal bearing structure is approximately simplified to the separate rotor structure as shown in Fig. 3b, where the oil-film supports are equivalent to four dynamic oil-film loads and several measuring points are respectively selected for the oil-film load reconstruction and the oil-film coefficient verification. As the oil-film damping and stiffness characteristics are actually functions of few structural parameters, such as the radial clearance, the absolute viscosity of the lubricant, the unbalance mass and the rotor speed, etc., in this paper, a wide range of rotor speeds from $\mathrm{R}=600 \mathrm{r} / \mathrm{min}$ ( $R=60 \omega / 2 \pi$ ) to $\mathrm{R}=2000 \mathrm{r} / \mathrm{min}$ is investigated to throw lights on the oil-film 
characteristics and 5 percent noise is mixed into the computed responses to simulate the measurement. In the following parts, we mainly take the details of the cases $\mathrm{R}=600 \mathrm{r} / \mathrm{min}$ and $\mathrm{R}=1000 \mathrm{r} / \mathrm{min}$ for example to illustrate the identification procedures with the other cases being similar.

Within the presented method, it is first required to obtain the structural unbalance responses for the load reconstruction. As an example, the simulated unbalance displacement responses at two measuring points are shown in Fig. 4 denoted by the red dotted lines. It is easy to see that the response curves disturb slightly at the beginning, which can be explained by the initial transient caused by time integration, and reach stable quickly, which is in line with the reality.
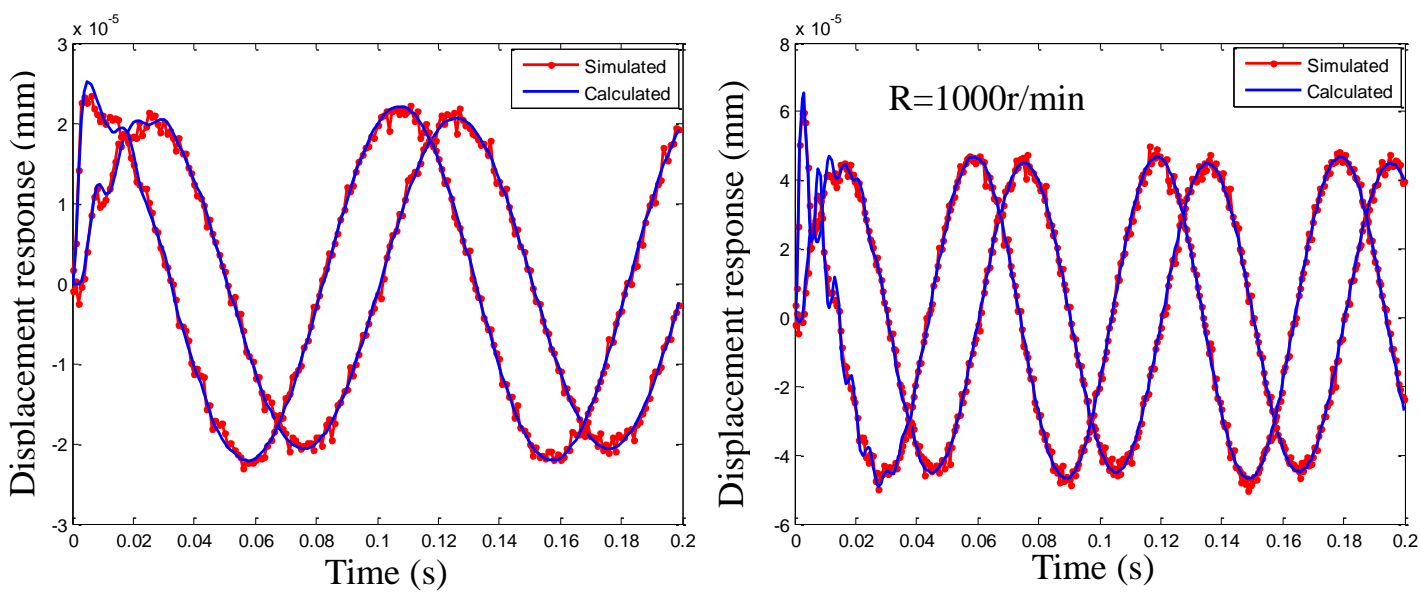

Fig. 4 Structural unbalance displacement responses

For the rotor structure shown in Fig. 3b, the Green's function method is implemented to realize the oil-film load reconstruction. Four displacement responses at different nodes (as shown in the figure) are measured and with the help of regularization, the oil-film loads are stably obtained and shown in Fig. 5. Obviously, they all have the harmonic forms, and there exist a regular $\pi / 2$ phase difference between the two components of oil-film loads. 

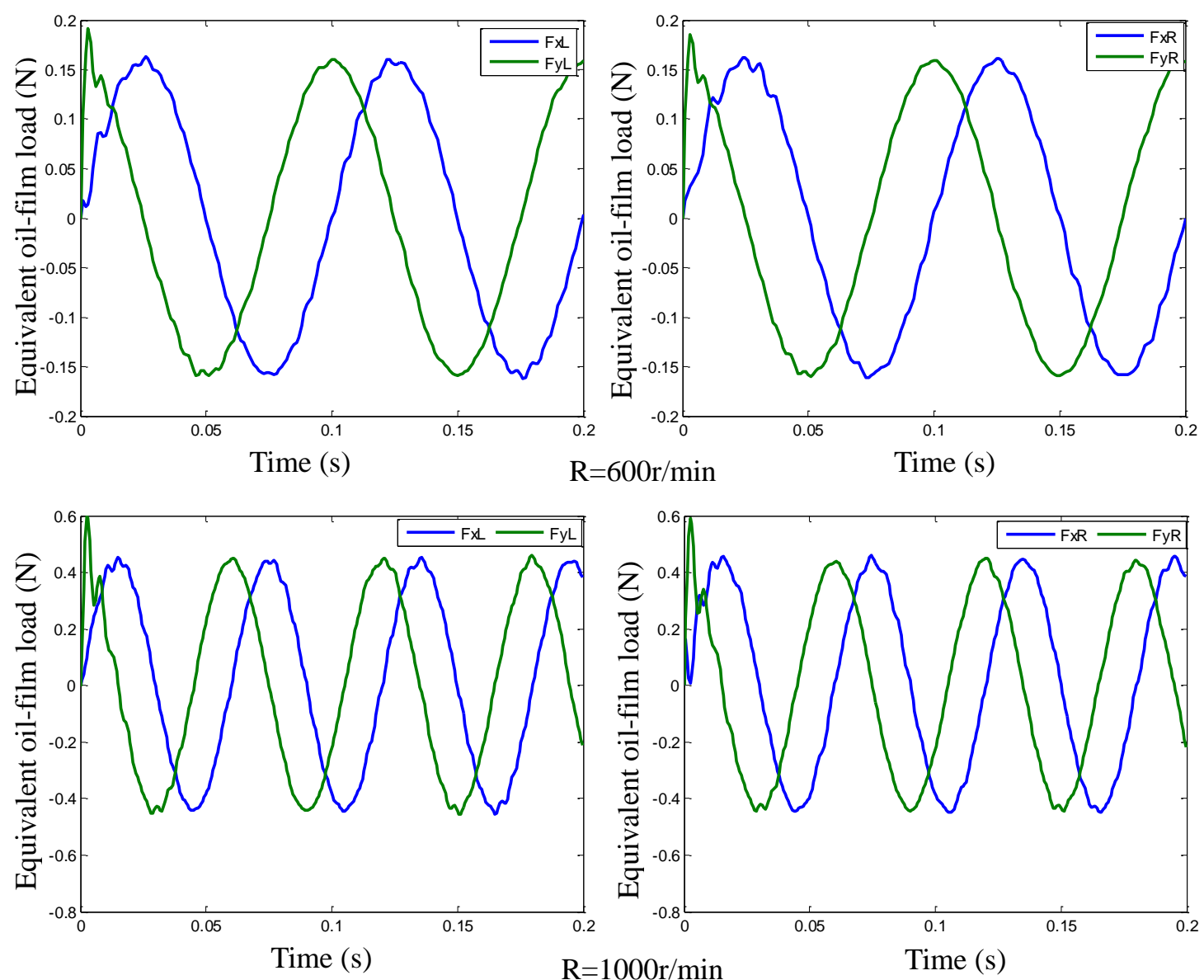

Fig. 5 The reconstructed oil-film loads

As the reconstruction precision of the equivalent oil-film loads has an essential and direct influence on the subsequent identification of oil-film coefficient, they are applied to Eq. (8) to numerically calculate structural responses at the measuring points and to verify the correctness of the loads. Shown in Fig. 4, the calculated responses which are represented by blue solid lines fit the previously measured ones pretty well, which ensures the precision of the reconstructed loads. Meanwhile, the unbalance displacement and velocity responses at the DOFs of the journal bearing supporting are also computed for the oil-film coefficient identification, which are plotted in Fig. 6. 

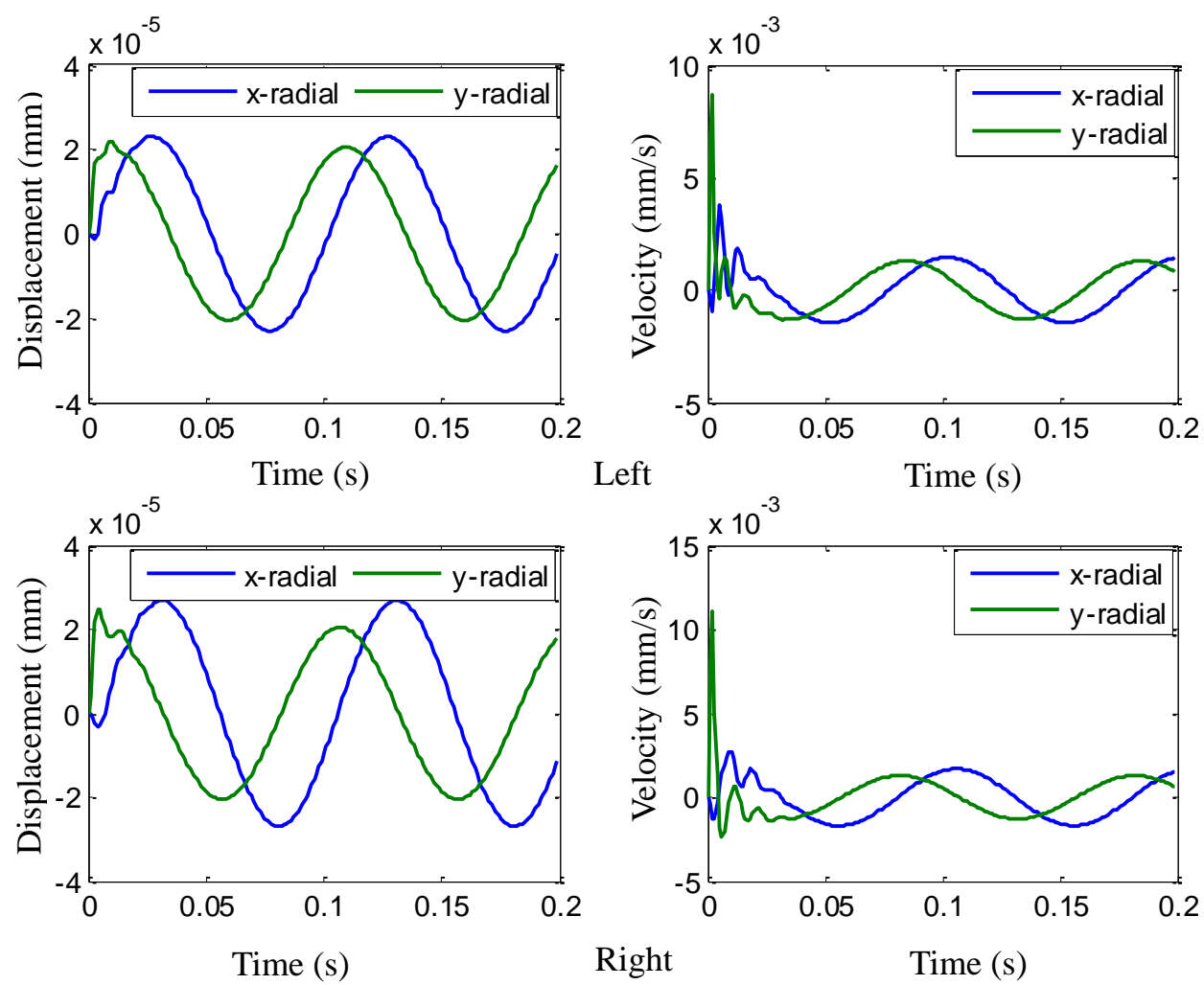

Fig. 6a The unbalance responses at the bearing supporting DOF with $\mathrm{R}=600 \mathrm{r} / \mathrm{min}$
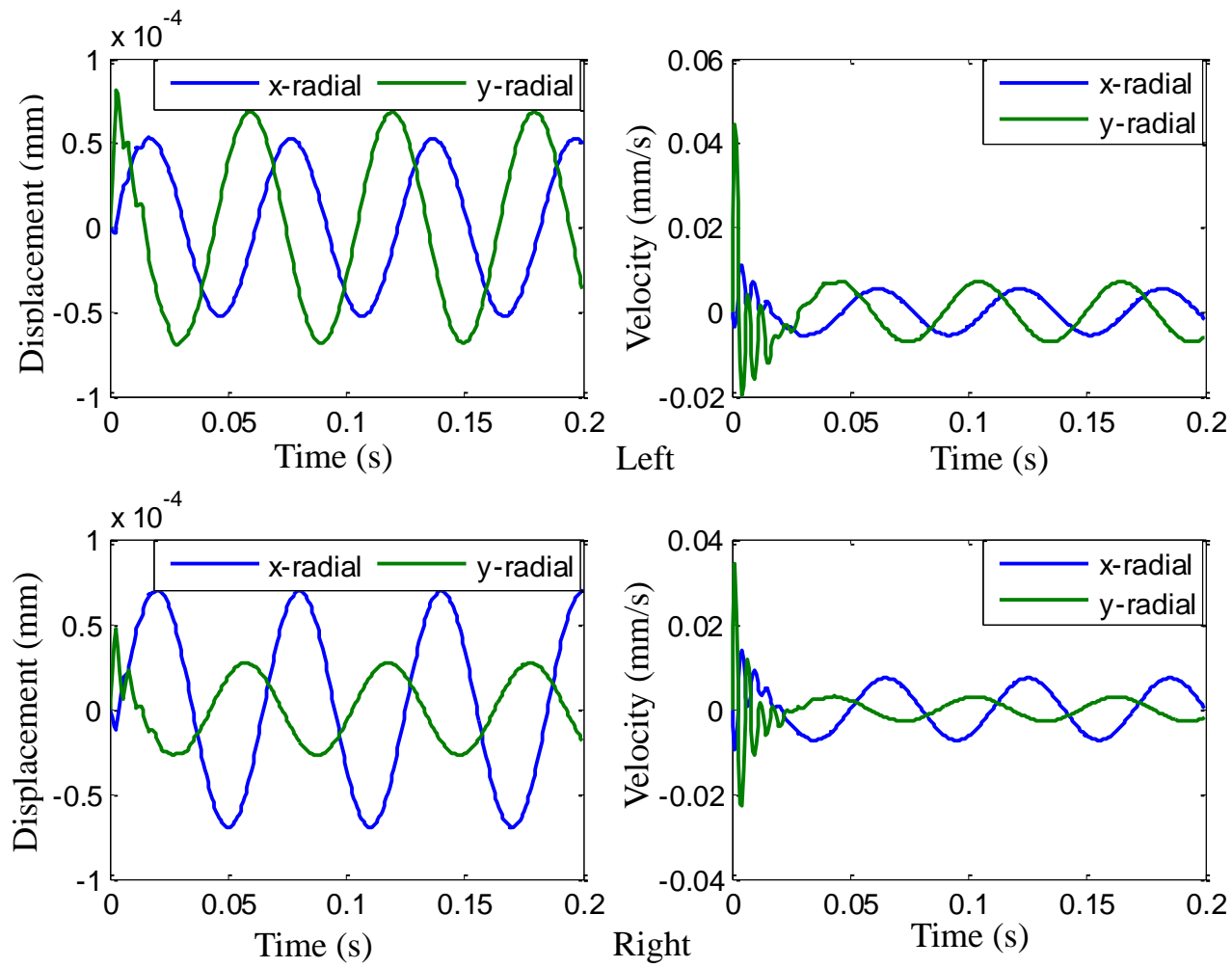

Fig. $6 \mathrm{~b}$ The unbalance responses at the bearing supporting DOF with $\mathrm{R}=1000 \mathrm{r} / \mathrm{min}$

Apparently, these responses disturb at the beginning as like the responses at other 
DOFs of the rotor (shown in Fig. 4). So, to avoid the adverse influence of the initial significant derivations of the responses on the identification precision of oil-film coefficients, the weighted residual integration method is conducted with the initial time for the integration being set $t_{0}=0.03 \mathrm{~s}$. The identified oil-film coefficients are then obtained and listed in Tab. 1. To investigate the relationships between the identified oilfilm damping and stiffness coefficients and the other structural parameters, a dimensionless quantity, the Sommerfeld number [29], which contains all the variables normally specified by the designer, is employed to describe oil-film coefficients. In this paper, we perform oil-film loads and coefficients identification under relatively stable rotor speeds (the assumption that the fluid is incompressible is applicable), and don't take into consider the change of temperature, which means the radial clearance and the viscosity of the lubricant are constant. So, the Sommerfeld number mainly depends on the rotor speed. In Fig. 7, a part relationship between the Sommerfeld number and the oil-film characteristic damping and stiffness are plotted. Easy to see, in this rotor speed range, the damping and stiffness coefficients generally increase with the growth of the Sommerfeld number, but the trend presents certain nonlinearity and complexity, which is in good agreement with the actual situation. To ultimately test the validity of the identified oil-film coefficients, the coefficients are finally applied to the rotor-journal bearing structure (Eq. (1)). The comparison between the measured and calculated $\mathrm{x}$ and y-radial displacements at the point selected in Fig. $3 b$ are shown for example in Fig. 8. The whole relative errors which are listed in the last column of Tab. 1, are 5.75 percent and 7.95 percent, respectively. The small errors indicate that the identified results are satisfactory and the proposed method is efficient. 

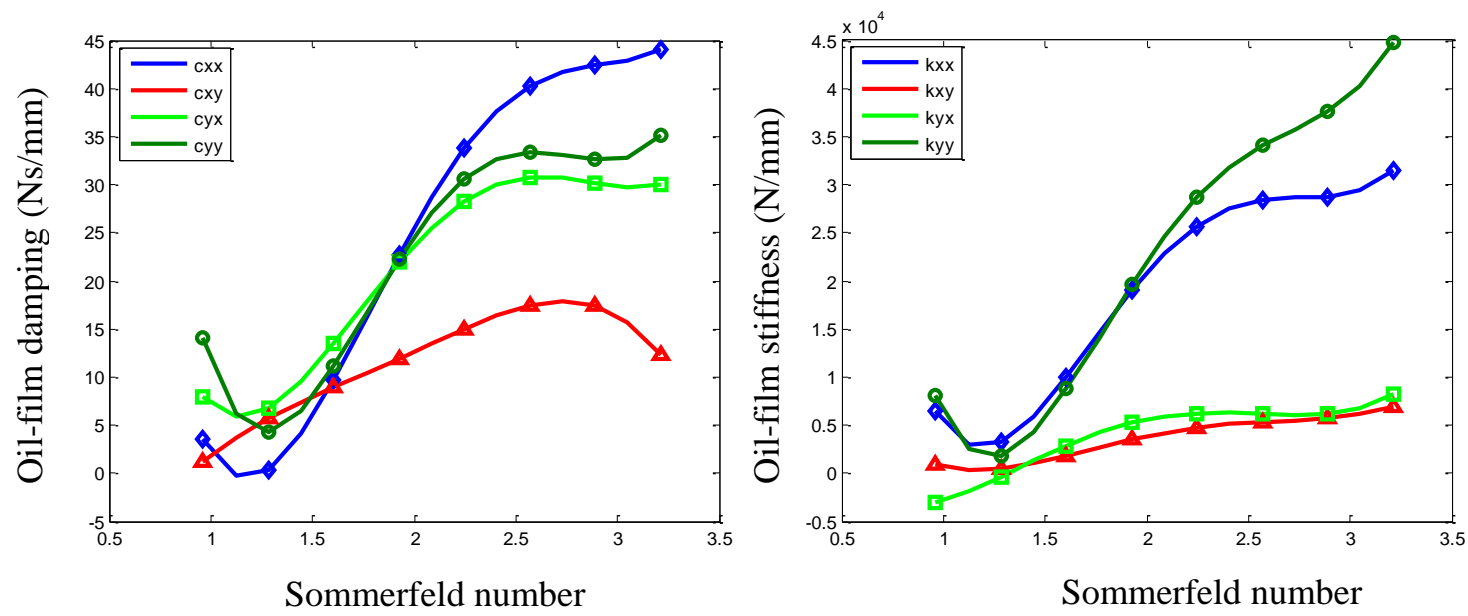

Fig. 7a Sommerfeld number effect on the left bearing oil-film characteristics
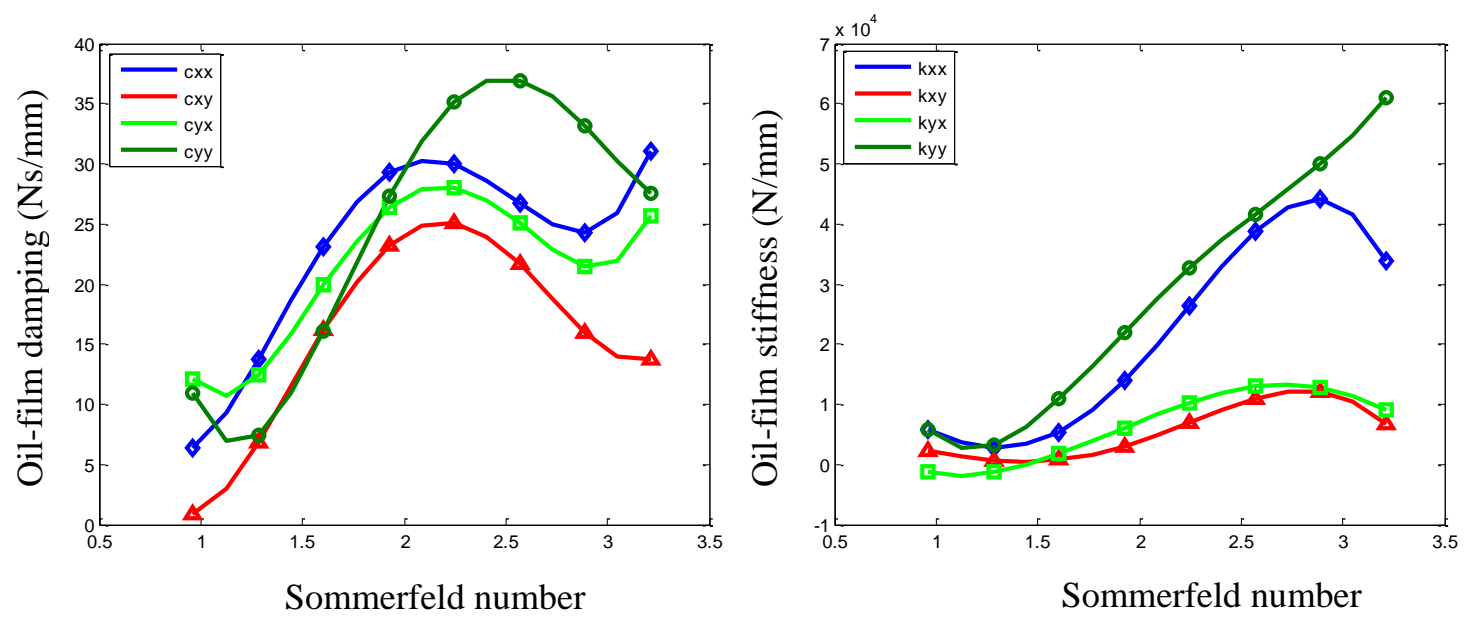

Fig. 7b Sommerfeld number effect on the right bearing oil-film characteristics
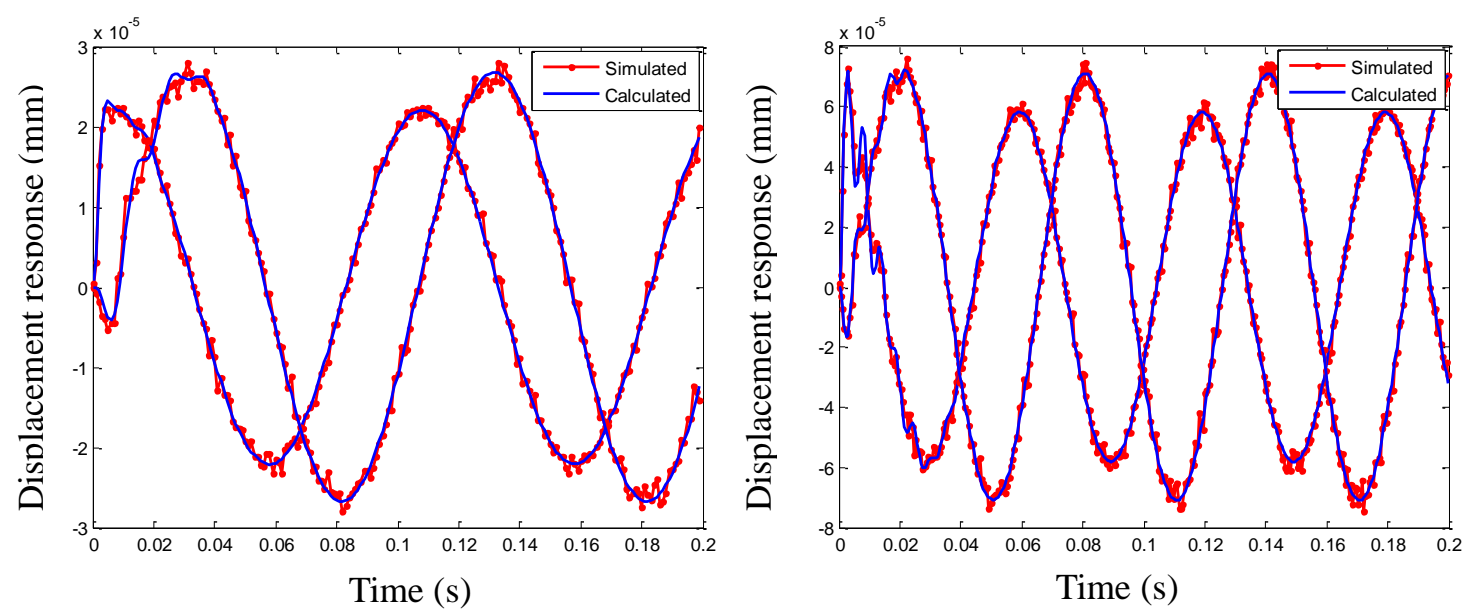

Fig. 8 Comparison between the measured responses and calculated ones 
Tab. 1 Identified oil-film coefficients under different angular speeds

\begin{tabular}{|c|c|c|c|c|c|c|c|c|c|c|}
\hline \multirow{2}{*}{\multicolumn{2}{|c|}{$\begin{array}{l}\text { Rotate speed } \\
\qquad(\mathrm{r} / \mathrm{min})\end{array}$}} & \multicolumn{4}{|c|}{ Stiffness $\left(\times 10^{4} \mathrm{~N} / \mathrm{mm}\right)$} & \multicolumn{4}{|c|}{ Damping (Ns/mm) } & \multirow{2}{*}{$\begin{array}{c}\text { Whole } \\
\text { relative } \\
\text { errors }(\%)\end{array}$} \\
\hline & & $k_{x x}$ & $k_{x y}$ & $k_{y x}$ & $k_{y y}$ & $c_{x x}$ & $c_{x y}$ & $c_{y x}$ & $c_{y y}$ & \\
\hline \multirow{2}{*}{600} & Left & 0.647 & 0.091 & -0.323 & 0.743 & 2.752 & 1.001 & 9.096 & 13.466 & \multirow{2}{*}{5.75} \\
\hline & Right & 0.541 & 0.222 & -0.191 & 0.612 & 7.040 & 0.675 & 13.000 & 10.116 & \\
\hline \multirow{2}{*}{800} & Left & 0.338 & 0.075 & 0.082 & 0.450 & 4.026 & 7.103 & 2.679 & 7.024 & \multirow{2}{*}{8.39} \\
\hline & Right & 0.336 & 0.069 & 0.073 & 0.216 & 11.118 & 8.133 & 8.762 & 10.982 & \\
\hline \multirow{2}{*}{1000} & Left & 0.863 & 0.089 & 0.093 & 0.532 & 4.031 & 4.979 & 17.236 & 6.038 & \multirow{2}{*}{7.95} \\
\hline & Right & 0.597 & 0.047 & 0.016 & 1.116 & 26.696 & 12.902 & 25.270 & 10.684 & \\
\hline \multirow{2}{*}{1200} & Left & 2.155 & 0.470 & 0.545 & 2.061 & 27.138 & 16.646 & 24.237 & 26.363 & \multirow{2}{*}{7.18} \\
\hline & Right & 1.278 & 0.222 & 0.324 & 2.326 & 28.390 & 26.342 & 23.184 & 29.290 & \\
\hline \multirow{2}{*}{1400} & Left & 2.448 & 0.435 & 0.945 & 2.939 & 31.044 & 13.454 & 22.976 & 30.547 & \multirow{2}{*}{8.38} \\
\hline & Right & 2.455 & 1.073 & 1.642 & 3.321 & 27.851 & 25.201 & 29.272 & 38.343 & \\
\hline \multirow{2}{*}{1600} & Left & 2.749 & 0.476 & 0.198 & 3.445 & 42.976 & 15.444 & 32.391 & 31.370 & \multirow{2}{*}{9.80} \\
\hline & Right & 4.360 & 0.580 & 0.867 & 3.816 & 28.513 & 18.993 & 23.255 & 32.959 & \\
\hline \multirow{2}{*}{1800} & Left & 2.986 & 0.631 & 0.833 & 3.655 & 40.409 & 19.386 & 31.246 & 33.867 & \multirow{2}{*}{7.24} \\
\hline & Right & 4.057 & 1.499 & 1.403 & 5.290 & 23.967 & 17.927 & 23.218 & 34.835 & \\
\hline \multirow{2}{*}{2000} & Left & 3.114 & 0.681 & 0.780 & 4.511 & 44.516 & 11.754 & 29.462 & 34.881 & \multirow{2}{*}{8.08} \\
\hline & Right & 3.480 & 0.604 & 0.896 & 6.014 & 30.973 & 13.292 & 25.187 & 27.361 & \\
\hline
\end{tabular}

\section{Conclusion}

A new method based on equivalent oil-film load reconstruction is proposed for the identification of oil-film characteristic coefficients, which plays a very essential role in monitoring and maintaining the stable work of the rotor-journal bearing system, and conducting system fault diagnosis etc. It approximates the oil-film supports of the journal bearings as several load boundary conditions on the easy modeling rotor structure, which effectively avoids the difficulty in modeling the complicated oil-film and is helpful to improve the identification precision. The loads are simply related to the oil-film stiffness and damping coefficients, and can be stably and accurately 
reconstructed by the Green's function method with regularization. With the well reconstructed oil-film loads, the weighted residual integration method based on least squares is conducted to identify the oil-film coefficients, which comprehensively utilizes the information of the reconstructed loads and calculated unbalance responses in the time domain. The process of the proposed method has been presented in detail in the example and satisfied results are obtained and tested, demonstrating that the method is valid and accurate.

On the other hand, it is still a laboratory identification technique with the aid of the finite element simulation, not a field technique. In the laboratory environment, the rotor can be relatively easy to model by finite element method, the unbalance mass, eccentricity, and initial phase of the rotor can be measured, and sufficient unbalance responses are available. And currently, we consider the simplified case without considering the fluid forces applied by impellers, seals etc. In the near future, complicated field cases and precise models are to be investigated.

\section{Acknowledgement}

This work is supported by the National Science Foundation of China (11572115), the independent research project of State Key Laboratory of Advanced Design and Manufacturing for Vehicle Body (51475003), the Changzhou City Science and Technology Support Program (CE20140027), and the graduate student research innovation project of Hunan province (CX2016B090).

\section{References}

[1] Tiwari R, Lees AW, Friswell MI. Identification of dynamic bearing parameters: a review. Shock Vib Digest 2004; 36(2): 99-124. 
http://dx.doi.org/10.1177/0583102404040173.

[2] Tønder K. Dimpled pivoted plane bearings: Modified coefficients. Tribol Int 2010; 43 (2010): 2303-2307. http://dx.doi.org/10.1016/j.triboint.2010.08.001.

[3] Chang SH, Chan CW, Jeng YR. Numerical analysis of discharge coefficients in aerostatic bearings with orifice-type restrictors. Tribol Int 2015; 90: 157-163. http://dx.doi.org/10.1016/j.triboint.2015.04.030.

[4] Rana1 NK, Gautam1 SS, Verma S, Rahmani F. On the stiffness and damping coefficients of constant flow valve compensated conical hydrostatic journal bearing with micropolar lubricant. Procedia Tech 2016; 23: 42-50.

http://creativecommons.org/licenses/by-nc-nd/4.0/

[5] Nicholas JC, Gunter EJ, Allaire PE. Stiffness and damping coefficients for the fivepad tilting-pad bearing. Tribol Trans 1979; 2(113): 113-124. http://dx.doi.org/10.1080/05698197908982907.

[6] Tieu AK, Qiu ZL. Identification of sixteen dynamic coefficients of two journal bearings from experimental unbalance responses. Wear 1994; 177: 63-69. http://dx.doi.org/10.1016/0043-1648(94)90118-X.

[7] Qiu ZL, Tieu AK. Identification of sixteen force coefficients of two journal bearings from impulse responses. Wear 1997; 212: 206-212. http://dx.doi.org/10.1016/S0043-1648(97)00154-3.

[8] Jiang GD, Hu H, Xu W, Jin ZW, Xie YB. Identification of oil film coefficients of large journal bearings on a full scale journal bearing test rig. Tribol Int 1997; 30(11): 789-793. http://dx.doi.org/10.1016/S0301-679X(97)00040-6.

[9] Tiwari R, Chakravarthy V. Simultaneous identification of residual unbalances and bearing dynamic parameters from impulse responses of rotor-bearing systems. Mech Syst Signal Pr 2006; 20: 1590-1614. http://dx.doi.org/10.1016/j.ymssp.2006.01.005.

[10]R. Tiwari, V. Chakravarthy, Simultaneous estimation of the residual unbalance and bearing dynamic parameters from the experimental data in a rotor-bearing system. Mech Mach Theory 2009; 44: 792-812. http://dx.doi.org/10.1016/j.mechmachtheory.2008.04.008. 
[11]Zhang YY, Xie YB, Qiu DM. Identification of linearized oil-film coefficients in a flexible rotor-bearing system, part I: model and simulation. J Sound Vib 1992; 152: 531-547. http://dx.doi.org/10.1016/0022-460X(92)90485-G.

[12]Zhao SX, Zhou H, Meng G, Zhu J. Experimental identification of linear oil-film coefficients using least-mean-square method in time domain. J Sound Vib 2005; 287: 809-825. http://dx.doi.org/10.1016/j.jsv.2004.12.011.

[13]Zhao SX, Dai XD, Meng G, Zhu J. An experimental study of nonlinear oil-film forces of a journal bearing. J Sound Vib 2005; 287: 827-843. http://dx.doi.org/10.1016/j.jsv.2004.11.034.

[14]Makowski R, Zimroz R. A procedure for weighted summation of the derivatives of reflection coefficients in adaptive Schur filter with application to fault detection in rolling element bearings. Mech Syst Signal Pr 2013; 38: 65-77. http://dx.doi.org/10.1016/j.ymssp.2012.05.005.

[15]Zhang XL, Yin ZW, Gao GY, Li Z. Determination of stiffness coefficients of hydrodynamic water-lubricated plain journal bearings. Tribol Int 2015; 85: 37-47. http://dx.doi.org/10.1016/j.triboint.2014.12.019.

[16] Wang WM, Li QH, Gao JJ, Yao JF, Allaire P. An identification method for damping ratio in rotor systems. Mech Syst Signal Pr 2016; 68-69: 536-554.

http://dx.doi.org/10.1016/j.ymssp.2015.05.023.

[17]Zhou J, Di L, Cheng CG, Xu YP, Lin ZL. A rotor unbalance response based approach to the identification of the closed-loop stiffness and damping coefficients of active magnetic bearings. Mech Syst Signal Pr 2016; 66-67: 665-678. http://dx.doi.org/10.1016/j.ymssp.2015.06.008.

[18]Zhou H, Zhao SX, H Xu, Zhu J. An experimental study on oil-film dynamic coefficients. Tribol Int 2004; 37: 245-253.

http://dx.doi.org/10.1016/j.triboint.2003.08.002.

[19]Synnegård E, Gustavsson R, Aidanpää JO. Influence of cross-coupling stiffness in tilting pad journal bearings for vertical machines. Int J Mech Sci 2016; 111-112: 43-54. http://dx.doi.org/10.1016/j.ijmecsci.2016.03.017.

[20] Kim YH, Yang BS, Tan ACC. Bearing parameter identification of rotor-bearing $-24-$ 
system using clustering-based hybrid evolutionary algorithm. Struct Multidisc Optim 2007; 33: 493-506. http://dx.doi.org/10.1007/s00158-006-0055-5.

[21] Meruane V, Pascual R. Identification of nonlinear dynamic coefficients in plain journal bearings. Tribol Int 2008; 41: 743-754. http://dx.doi.org/10.1016/j.triboint.2008.01.002.

[22] Kim TH, Andrés LS. Analysis of advanced gas foil bearings with piecewise linear elastic supports. Tribol Int 2007; 40: 1239-1245. http://dx.doi.org/10.1016/j.triboint.2007.01.022.

[23]Nelson HD. A Finite Rotating Shaft Element Using Timoshenko Beam Theory. J Mech Des 1980; 102: 793-803. http://dx.doi.org/10.1115/1.3254824.

[24]Li K, Liu J, Han X, Sun XS, Jiang C. A novel approach for distributed dynamic load reconstruction by space-time domain decoupling. J Sound Vib 2015; 348(21): 137-145. http://dx.doi.org/10.1016/j.jsv.2015.03.009.

[25]Liu J, Sun XS, Han X, Jiang C, Yu DJ. A novel computational inverse technique for load identification using the shape function method of moving least square fitting. Comput Struct 2014; 144: 127-137. http://dx.doi.org/10.1016/j.compstruc.2014.08.002.

[26]Liu J, Meng XH, Jiang C. Time-domain Galerkin method for dynamic load identification. Int J Numer Meth Eng 2016; 6(105): 620-640. http://dx.doi.org/10.1002/nme.4991.

[27] Sun XS, Liu J, Han X, Jiang C, Chen R. A new improved regularization method for dynamic load identification. Inverse Proble Sci En 2014; 22(7): 1062-1076. http://dx.doi.org/10.1080/09205063.2013.854353.

[28] Golub GH, Heath M, Wahba G. Generalized cross-validation as a method for choosing a good ridge parameter. Technimetrics 1979; 21: 215-223. http://dx.doi.org/10.1080/00401706.1979.10489751.

[29] Golub GH, Heath M, Wahba G. Generalized cross-validation as a method for choosing a good ridge parameter. Technimetrics 1979; 21:215-223. http://dx.doi.org/10.1080/00401706.1979.10489751.

[30] Samantaray AK, Dasgupta SS, Bhattacharyya R. Sommerfeld effect in rotationally $-25-$ 
symmetric planar dynamical systems. Int J Eng Sci 2010; 48(1): 21-36.

http://dx.doi:10.1016/j.ijengsci.2009.06.005 